\title{
Barriers to Care at High Volume Centers in Hepatic Surgery
}

\section{Gregory C Wilson, Jeffrey M Sutton, Ralph Cutler Quillin III, Koffi Wima, Dennis J Hanseman, Jeffrey J Sussman, Syed A Ahmad, Shimul A Shah and Daniel E Abbott*}

Department of Surgery, University of Cincinnati Medical Center, Cincinnati Research in Outcomes and Safety in Surgery (CROSS), Cincinnati, Ohio, USA

\begin{abstract}
Purpose

High Volume Centers (HVC) have demonstrated superior outcomes with complex surgery. Understanding factors in accessing HVC may reduce disparities in quality and improve patient outcomes. Methods

The University Health systems Consortium (UHC) database identified 4,147 patients undergoing hepatic lobectomy from 2009-2011. Centers were stratified into tertiles based on the annual number of procedures performed. Geographic analysis was performed with maptitude geographic information software. Logistic regression was used to determine how patient demographics contributed to receiving care at a HVC.

Results

HVC represented $12 \%$ of the centers performing lobectomy over the study time period. Median distance for patients to HVC was 123.4 miles. $76 \%$ of patients with the worst severity of illness score did not utilize a high volume center. Independent predictors for not accessing a HVC included black race (OR=0.673, 95\% $\mathrm{Cl}=0.525-0.862 ; \mathrm{p}=0.002)$, residing further from HVC (OR=0.766, $95 \% \mathrm{Cl}=0.730-0.804 ; p<0.001$; per 100 mile increment), worsening severity of illness and patient disease (OR=0.782, 95\% $\mathrm{Cl}=0.703-0.870 ; \mathrm{p}<0.001)$, lower Socioeconomic Status (SES) $(\mathrm{OR}=0.766,95 \% \mathrm{Cl}=0.617-0.952 ; \mathrm{p}=0.016$ for $\mathrm{Q} 1$ and $\mathrm{OR}=0.766$, $95 \% \mathrm{Cl}=0.620-0.946 ; \mathrm{p}=0.013$ for $\mathrm{Q} 2)$ and uninsured patients (OR=0.428, 95\% Cl=0.242-0.756; $p=0.004)$.

\section{Conclusion}

Patient-specific disparities exist in accessing HVC for hepatic lobectomy. As regionalization of complex surgery increases, these
\end{abstract}

*Corresponding author: Daniel E Abbott, Department of Surgery, University of Cincinnati Medical Center, Cincinnati Research in Outcomes and Safety in Surgery (CROSS), 231 Albert Sabin Way, ML 0558, Cincinnati, Ohio, USA, Tel: +1 5135588900; E-mail: abbottdl@ucmail.uc.edu

Citation: Wilson GC, Sutton JM, Quillin III RC, Wima K, Hanseman DJ, et al. (2015) Barriers to Care at High Volume Centers in Hepatic Surgery. J Cancer Biol Treat 2: 004.

Received: December 16, 2014; Accepted: August 21, 2015; Published: September 04, 2015 factors must be recognized as barriers to receiving care at experiences centers.

\section{Introduction}

One decade ago, a landmark study investigating the effect of hospital volume on surgical mortality demonstrated that increasing surgical volume was associated with improved perioperative mortality [1]. This report including several others started a national trend in not only evaluating the effect of center volume on surgical outcomes but also advocating for patients to be directed to centers with high procedural volume [2-7]. Since then, numerous reports repeatedly demonstrated improved outcomes after complex surgical procedures including hepatic resection $[8,9]$. A recent report on the nationwide inpatient sample from 1995-2009 demonstrated that the increased focus on surgical outcomes and regionalization of complex care has resulted in improved surgical outcomes not only at High Volume Centers (HCV), but across the board at all centers [9]. Despite these improvements disparities still exist between HVC and all other centers [9].

Given this increased emphasis on centralization or regionalization of complex care and the documented improved outcomes at HVC, the goal of this current study is to evaluate patient factors associated with access (or the inability to access) specialized centers capable of performing complex surgical interventions such as hepatectomy. The number of specialized centers in the country is limited, and directing all patients in need of complex intervention to one of these centers may be associated with significant barriers for which some patients might be unable to overcome. We hypothesize that patients residing outside of urban areas with specialized centers would be less likely to access HVC.

\section{Methods}

This study was approved by and conducted in accordance with the University of Cincinnati's Institutional Review Board.

The University Health Consortium (UHC) Clinical Database/Resource Manager (CDB/RM) is an administrative database that has found recent utilization in health services research [10-12]. This administrative database has been demonstrated to capture accurate clinical data in the setting of complex surgical care [13]. The UHC CDB/RM was queried to identify all patients undergoing hepatectomy from 2009 through 2011. Patients were identified based on ICD-9 procedural codes and were limited to adult patients (18 years and older) undergoing hepatic lobectomy (ICD-9 procedural code 50.3).

Centers performing hepatic lobectomy were identified by a unique database identifier. Annual center volume for each unique center was based on annual case volume; three center groups were created based on such case volume. The centers were divided into tertiles, in which the High Volume Centers (HCV) were the tertile with highest annual case volume, Middle Volume Centers (MCV) were the centers in the middle tertile and Low Volume Centers (LVC) consisted of the remainder of the centers. This method for defining and evaluating center volume has been previously described $[1,14,15]$. 
Citation: Wilson GC, Sutton JM, Quillin III RC, Wima K, Hanseman DJ, et al. (2015) Barriers to Care at High Volume Centers in Hepatic Surgery. J Cancer Biol Treat 2: 004 .

- Page 2 of 4 •

Patient Socioeconomic Status (SES) was based on patient geographic factors utilizing United States census data. The SES for each patient is based on a combination of household income, housing value, employment status, and education level, as previously described $[16,17]$. Based on this summary score, patients were categorized into quintiles with Quintile 1(Q1) representing patients with the lowest SES, while Quintile 5 (Q5) consisted of people with the highest SES. Geographical analysis was performed using Maptitude Geographic Information System software (Version 2013, Caliper Corporation, Newton, MA). Patient proximity to centers was determined using postal zip code information. Patient's overall disease burden and comorbid medical condition severity were stratified based on the 3M All Patient Refined (APR) Severity of Illness (SOI) [18]. Patients receive a score of 1-4.

Statistical analysis was performed using SAS version 9.3 statistical software (SAS Institute, Inc., Cary, NC, USA). Categorical variables were analyzed using chi-square analysis while continuous variables were evaluated using wilcoxon rank-sum tests. Multivariate analysis was performed using logistic regression analysis. The final multivariate model included variables that were statistically significant on univariate analysis. The primary outcome for multivariate analysis was utilization of a HVC for hepatic lobectomy. Statistical significance was defined as a p value less than 0.05 for the given statistical method.

\section{Results}

From 2009-2011, 4,147 patients underwent hepatic lobectomy in the United States at one of the medical centers in the UHC CDB/RM. Patient demographics are listed in table 1. These patients underwent hepatic lobectomy at 109 medical centers across the nation. Center specific details are listed in table 2 including annual case volume ranges for each tertile, number of centers in each group, and overall case volume. HVC represent $12 \%$ of all the centers included in this study. Median distance from patient residence to HVC was 123.4 miles $(\mathrm{IQR}=32.5-223.8)$. Median distance from patient residence to LVC or MVC was25.7 miles (IQR = 9.6-66.6).

\begin{tabular}{|l|l|}
\hline \multicolumn{1}{|c|}{ Patient Characteristic } & \multicolumn{1}{c|}{ Median (IQR) or \% (n) } \\
\hline Age (years) & $57(47-67)$ \\
\hline Sex-(males) & $49.9 \%(2,073)$ \\
\hline Race & $73.1 \%(3,032)$ \\
\hline White & $9.7 \%(404)$ \\
\hline Black & $17.1 \%(711)$ \\
\hline Other & \multicolumn{2}{|l|}{} \\
\hline Socioeconomic Status & $19.6 \%(811)$ \\
\hline Q1-Lowest & $19.6 \%(812)$ \\
\hline Q2 & $19.6 \%(814)$ \\
\hline Q3 & $19.6 \%(811)$ \\
\hline Q4 & $19.7 \%(815)$ \\
\hline Q5-Highest & $25.7(9.6-66.6)$ \\
\hline $\begin{array}{l}\text { Proximtiy to Low or Medium Volume } \\
\text { Center (miles) }\end{array}$ & $123.4(32.5-223.8)$ \\
\hline Proximity to High Volume Center (miles) & \multicolumn{2}{|l}{} \\
\hline Severity of Illness & $65.3 \%(2,711)$ \\
\hline 2 & $25.1 \%(1,039)$ \\
\hline 3 & $9.6 \%(397)$ \\
\hline 4 & \\
\hline
\end{tabular}

\begin{tabular}{|l|l|}
\hline \multicolumn{2}{|l|}{ Insurance Status } \\
\hline Private & $52.4 \%(2,171)$ \\
\hline Government & $43.0 \%(1,785)$ \\
\hline Self Pay & $2.3 \%(96)$ \\
\hline Uninsured & $2.0 \%(81)$ \\
\hline Charity & $0.3 \%(14)$ \\
\hline
\end{tabular}

Table 1: Patient Demographics for Patients undergoing Hepatic Lobectomy from 2009-2011.

\begin{tabular}{|l|l|l|l|}
\hline Center Characteristic & $\begin{array}{l}\text { Low Volume } \\
\text { Centers }\end{array}$ & $\begin{array}{l}\text { Medium Volume } \\
\text { Centers }\end{array}$ & $\begin{array}{l}\text { High Volume } \\
\text { Centers }\end{array}$ \\
\hline Case Range & $1-16$ & $17-26$ & $27-78$ \\
\hline Total Number of Cases & 1,350 & 1,372 & 1,441 \\
\hline Number of Centers & 74 & 22 & 13 \\
\hline
\end{tabular}

Table 2: Center Volume Analysis for Centers Performing Hepatic Lobectomy from 2009-2011.

Note: Center volume was based on annual case volume performed at each center in the database. Centers were then divided into tertiles based on the annual case volume. Low volume centers represent the tertile of centers with the lowest annual case volume, while medium volume centers represents the middle tertile, etc. Case range is the number of cases performed per year. Total number of cases represents the total number of operations performed at a center in one of the three categories.

Next we examined the differences between patient populations that utilized each of the different center categories. Characteristics for each patient population broken down by center volume category are presented in table 3. Patients at HVC were less likely to be black $(7.3 \%)$ compared to LVC (10.8\%) and MVC (11.3\%). There was no difference in the proximity to LVC or MVC among the patients however patients undergoing surgery at HVC were much closer to a HVC (median=60.2 miles, $\mathrm{IQR}=16.4$-183.3) compared to patients at $\mathrm{LVC}$ (median=151.3, $\mathrm{IQR}=65.5-258$ ) or MVC (median=144.3, IQR=55.6-226.9). Patients at $\mathrm{HVC}$ also had lower overall severity of illness $(\mathrm{p}<0.001$ compared to LVC and MVC patients). More patients at HVC had private insurance $(55.9 \%)$ as primary payer and were in the highest SES quintile (22.9\%), when compared to patients at LVC and MVC.

Multivariate regression analysis was then performed to evaluate independent risk factors associated with utilizing or not utilizing HVC. The findings of our final model are depicted in figure 1. Independent risk factors that were associated with increased utilization of HVC included: primary payer status as self pay $(\mathrm{OR}=1.845,95 \% \mathrm{CI}=1.169-2.912 ; \mathrm{p}=0.009)$ and "other" race category $(\mathrm{OR}=1.367,95 \% \mathrm{CI}=1.147-1.629 ; \mathrm{p}=0.001)$. Factors that were associated with not utilizing HVC included: black race $(\mathrm{OR}=0.673,95 \%$ $\mathrm{CI}=0.525-0.862 ; \mathrm{p}=0.002)$, residing further from HVC $(\mathrm{OR}=0.766$, 95\% CI $=0.730-0.804 ; \mathrm{p}<0.001$; per 100 mile increment), worsening severity of illness and patient disease $(\mathrm{OR}=0.782, \quad 95 \%$ $\mathrm{CI}=0.703-0.870 ; \quad \mathrm{p}<0.001), \quad$ lower $\quad \mathrm{SES} \quad(\mathrm{OR}=0.766, \quad 95 \%$ $\mathrm{CI}=0.617-0.952 ; \mathrm{p}=0.016$ for $\mathrm{Q} 1$ and $\mathrm{OR}=0.766,95 \% \mathrm{CI}=0.620-0.946$; $\mathrm{p}=0.013$ for $\mathrm{Q} 2)$ and uninsured patients $(\mathrm{OR}=0.428,95 \%$ $\mathrm{CI}=0.242-0.756 ; \mathrm{p}=0.004$ ).

\section{Discussion}

Regionalization has been a developing trend in medicine focused on directing patients in need of complex medical care to highly specialized centers. The goal of this movement is to improve patient outcomes and avoid unnecessary morbidity and mortality that has been associated with less specialized centers. In this current study, a large, national cohort of patients undergoing hepatic lobectomy was evaluated. The focus of this study was to evaluate factors associated 
Citation: Wilson GC, Sutton JM, Quillin III RC, Wima K, Hanseman DJ, et al. (2015) Barriers to Care at High Volume Centers in Hepatic Surgery. J Cancer Biol Treat 2: 004 .

- Page 3 of $4 \cdot$

\begin{tabular}{|c|c|c|c|c|}
\hline Patient Characteristic & Low Volume Centers & Medium Volume Centers & High Volume Centers & $\mathbf{p}^{*}$ \\
\hline Age (years) & $57(47-67)$ & $58(48-68)$ & $56(46-66)$ & 0.026 \\
\hline Sex-(males) & $50.6 \%(679)$ & $47.8 \%(653)$ & $51.5 \%(741)$ & 0.148 \\
\hline Race & & & & $<0.001$ \\
\hline White & $71.8 \%(964)$ & $75.5 \%(1031)$ & $72.1 \%(1037)$ & \\
\hline Black & $10.8 \%(145)$ & $11.3 \%(154)$ & $7.3 \%(105)$ & \\
\hline Other & $17.4 \%(234)$ & $13.3 \%(181)$ & $20.6 \%(296)$ & \\
\hline Socioeconomic Status & & & & $<0.001$ \\
\hline Q1-Lowest & $22.5 \%(302)$ & $19.5 \%(266)$ & $16.9 \%(243)$ & \\
\hline Q2 & $19.8 \%(266)$ & $21.2 \%(290)$ & $17.8 \%(256)$ & \\
\hline Q3 & $20.4 \%(274)$ & $18.1 \%(247)$ & $20.4 \%(293)$ & \\
\hline Q4 & $19.3 \%(259)$ & $20.1 \%(275)$ & $19.3 \%(277)$ & \\
\hline Q5-Highest & $16.0 \%(215)$ & $19.8 \%(270)$ & $22.9 \%(330)$ & \\
\hline Proximtiy to Low or Medium Volume Center (miles) & $21.4(8.3-55.8)$ & $30.7(10.7-70.3)$ & $26.6(10.4-71.5)$ & 0.289 \\
\hline Proximity to High Volume Center (miles) & $151.3(65.5-258.0)$ & $144.3(55.6-226.9)$ & $60.2(16.4-183.3)$ & $<0.001$ \\
\hline Severity of IIIness & & & & $<0.001$ \\
\hline 2 & $62.2 \%(836)$ & $63.9 \%(873)$ & $69.7 \%(1002)$ & \\
\hline 3 & $27.1 \%(364)$ & $24.5 \%(335)$ & $23.6 \%(340)$ & \\
\hline 4 & $10.6 \%(143)$ & $11.6 \%(158)$ & $6.7 \%(96)$ & \\
\hline Insurance Status & & & & $<0.001$ \\
\hline Private & $48.3 \%(648)$ & $52.6 \%(719)$ & $55.9 \%(804)$ & \\
\hline Government & $47.1 \%(633)$ & $42.7 \%(583)$ & $39.6 \%(569)$ & \\
\hline Self Pay & $2.0 \%(27)$ & $1.7 \%(23)$ & $3.2 \%(46)$ & \\
\hline Uninsured & $2.2 \%(30)$ & $2.6 \%(35)$ & $1.1 \%(16)$ & \\
\hline Charity & $0.4 \%(5)$ & $0.4 \%(6)$ & $0.2 \%(3)$ & \\
\hline
\end{tabular}

Table 3: Patient Characteristics by Center Volume.

${ }^{*} p$-values represent the univariate logistic regression analysis with the primary outcome being going to high volume center for hepatic lobectomy

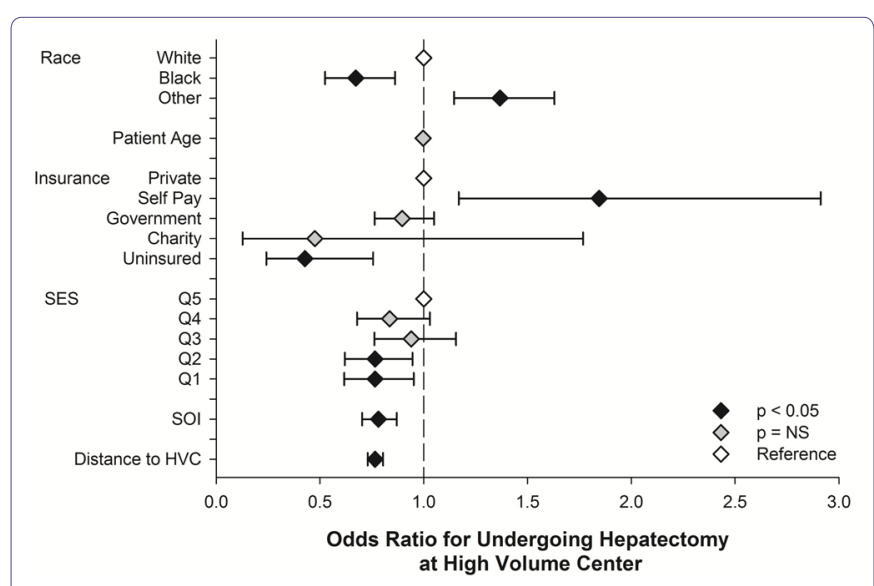

Figure 1: Multivariate analysis of factors associated with undergoing hepatic lobectomy at a high volume center. Only factors found to be significantly on univariate analysis were included in the final multivariate model of factors associated with accessing care at a high volume center. Odds ratios with $95 \%$ confidence intervals are depicted in the figure. SES-socioeconomic status quintile Q5 represents patients in the highest socioeconomic quintile, while Q1 represents patients in the lowest socioeconomic quintile. SOI-severity of illness as described in the methods section. Higher SOI scores represent worse overall disease state or progression. Distance to High Volume Center $(\mathrm{HCV})$ is the distance from the patient to the closest HVC.

with utilizing specialty centers and any potential barriers that may exist. This study adds to the fund of information regarding potential barriers to receiving care in a system where regionalization of complex care is prioritized $[9,19]$. We evaluated patient-specific barriers in accessing these specialized centers using logistic regression and found that several disparities do exist in the utilization of HVC for hepatic lobectomy. The disparities identified in this study included patient race, SES, geographic location, payer status, as well as disease severity and medical co-morbidities.

The UHC dataset provided a unique patient dataset that allowed us to analyze demographic, socioeconomic, and geographic factors and their role in center selection. One of the strengths of this study is the use of both patient demographics and geographic location in evaluating barriers to accessing HVC. Because HVC represented only $12 \%$ of all centers in this dataset, this small number of centers creates a geographical barrier for access. The median distance for patients to one of these centers is over 100 miles. Not surprisingly, our analysis demonstrates that the further someone lives from a HVC, the less likely they are to access a HVC for care. Additional barriers identified in this analysis include patient race, primary payer status, socioeconomic status, and severity of illness. Perhaps one of the more alarming findings of this study was that the sickest of patients were some of the least likely patients to access care at a HVC. In this patient population, $76 \%$ of patients with the worst severity of illness score went to either a LVC or MVC.

This study has its limitations. The retrospective nature limits any causal relationship between patient factors and outcomes. Additionally, the use of an administrative database limits our reporting of outcomes to determine if receiving care at a HVC does in fact incur improved results in the current health care environment. However numerous previous publications have continued to demonstrate improved outcomes at high volume, specialized centers. 
Citation: Wilson GC, Sutton JM, Quillin III RC, Wima K, Hanseman DJ, et al. (2015) Barriers to Care at High Volume Centers in Hepatic Surgery. J Cancer Biol Treat 2: 004 .

Also, the centers contained in this data are likely skewed towards tertiary academic centers which would likely be the benefactor of a system in which regionalization is emphasized.

\section{Conclusion}

In conclusion, this study represents an evaluation of potential barriers patients might face in an increasingly regionalized medical system. This study demonstrates that rural, poor, minority or sickest patients are at the highest risk for not accessing the most experienced centers, and that there should be built-in measures to address disparities that these high-risk patients may have in a system focused on regionalized care. Future policy aimed at directing patients with complex diseases to specialized centers must consider these data carefully in order to not increase access disparities.

\section{References}

1. Birkmeyer JD, Siewers AE, Finlayson EV, Stukel TA, Lucas FL, et al. (2002) Hospital volume and surgical mortality in the United States. N Engl J Med 346: 1128-1137.

2. Dimick JB, Cowan JA Jr, Knol JA, Upchurch GR Jr (2003) Hepatic resection in the United States: indications, outcomes, and hospital procedural volumes from a nationally representative database. Arch Surg 138: 185-191.

3. Finlayson EV, Goodney PP, Birkmeyer JD (2003) Hospital volume and operative mortality in cancer surgery: a national study. Arch Surg 138: 721-725.

4. Begg CB, Cramer LD, Hoskins WJ, Brennan MF (1998) Impact of hospital volume on operative mortality for major cancer surgery. JAMA 280: 17471751.

5. Glasgow RE, Showstack JA, Katz PP, Corvera CU, Warren RS, et al. (1999) The relationship between hospital volume and outcomes of hepatic resection for hepatocellular carcinoma. Arch Surg 134: 30-35.

6. Choti MA, Bowman HM, Pitt HA, Sosa JA, Sitzmann JV, et al. (1998) Should hepatic resections be performed at high-volume referral centers? J Gastrointest Surg 2: 11-20.

7. Milstein A, Galvin RS, Delbanco SF, Salber P, Buck CR Jr (2000) Improving the safety of health care: the leapfrog initiative. Eff Clin Pract 3: 313-316.
8. Finks JF, Osborne NH, Birkmeyer JD (2011) Trends in hospital volume and operative mortality for high-risk surgery. N Engl J Med 364: 2128-2137.

9. Colavita PD, Tsirline VB, Belyansky I, Swan RZ, Walters AL, et al. (2014) Regionalization and outcomes of hepato-pancreato-biliary cancer surgery in USA. J Gastrointest Surg 18: 532-541.

10. About UHC, 2013.

11. Salvalaggio PR, Dzebisashvili N, MacLeod KE, Lentine KL, Gheorghian A et al. (2011) The interaction among donor characteristics, severity of liver disease, and the cost of liver transplantation. Liver Transpl 17: 233-242.

12. Wilson GC, Cutler Quillin R 3rd, Sutton JM, Wima K, Shaw JJ, et al. (2015) Factors related to readmission after major elective surgery. Dig Dis Sci 60: 47-53.

13. Sutton JM, Hayes AJ, Wilson GC, Quillin RC 3rd, Wima K, et al. (2014) Validation of the University Health System Consortium administrative dataset: concordance and discordance with patient-level institutional data. J Surg Res 190: 484-490.

14. Singla A, Simons J, Li Y, Csikesz NG, Ng SC, et al. (2009) Admission volume determines outcome for patients with acute pancreatitis. Gastroenterology 137: 1995-2001.

15. Macomber CW, Shaw JJ, Santry H, Saidi RF, Jabbour N, et al. (2012) Centre volume and resource consumption in liver transplantation. HPB (Oxford) 14: 554-559.

16. Birkmeyer NJ, Gu N, Baser O, Morris AM, Birkmeyer JD (2008) Socioeconomic status and surgical mortality in the elderly. Med Care 46: 893-899.

17. Quillin RC 3rd, Wilson GC, Wima K, Hohmann SF, Sutton JM, et al. (2014) Neighborhood level effects of socioeconomic status on liver transplant selection and recipient survival. Clin Gastroenterol Hepatol 12: 1934-1941.

18. $3 M$ Health Information Systems (2013) $3 M^{T M}$ APR DRG Software-Account for severity and risk among all patients.

19. Liu JH, Zingmond DS, McGory ML, SooHoo NF, Ettner SL, et al. (2006) Disparities in the utilization of high-volume hospitals for complex surgery. JAMA 296: 1973-1980. 